\title{
Benefits of bloodletting procedure among Bad-mKhris-mGo-Nad patients: a prospective observational study
}

\author{
Karma Ugyen ${ }^{1}$, Karma Tenzin², Tempa Gyeltshen ${ }^{3}$ \\ ${ }^{I}$ National Traditional Medicine Hospital, Kawangjangsa, Thimphu, Bhutan \\ ${ }^{2}$ Faculty of Postgraduate Medicine, Khesar Gyalpo University of Medical Sciences of Bhutan, Thimphu, Bhutan \\ ${ }^{3}$ Faculty of Traditional Medicine, Khesar Gyalpo University of Medical Sciences of Bhutan, Thimphu, Bhutan
}

\begin{abstract}
Introduction: Bad-mKhris-mGo-Nad is a type of headache caused by imbalance of bile and phlegm humor. In gSo-ba-Rig-pa, headache is categorized in two categories such as primary headaches and secondary headaches. In total there are 14 types of headaches and Bad-mKhris-mGoNad is one among the principal headaches. The gSo-ba-Rig-pa based bloodletting therapy for Bad-mKhris-mGo-Nad has been practiced by the traditional medicine practitioners since the inception of $g S o-b a-R i g-p a$ more than 2500 years ago. Bloodletting is also believed to be one of the most important and effective therapy in the treatment regime of gSo-ba-Rig-pa. The Subsequent Tantra (last tantra) states that the bloodletting is the best remedy for all types of bile and blood-related disorders. This study was conducted to observe the effectiveness of bloodletting procedure among diagnosed cases of Bad-mKhris-mGo-Nad. Methods: It was an observational prospective study conducted at the National Traditional Medicine Hospital, Kawangjansa, Thimphu. The three main parameters measured were frequency, duration, and severity of the headaches. The samples consisted of 31 patients with 23 females and 8 males. Data collection was carried out over the span of seven months (June - December, 2018) Results: The association test between before and after 10 days, before and after 30 days of bloodletting was conducted on 30 patients by using Mid-Point test in Open Epi and the result was observed to be statistically significant at $p<0.05$. Conclusions: This study shows that the bloodletting therapy on Tshoksang reduces the pain duration and frequencies of headaches. Moreover, it was also established to be effective in reducing the severity of the headache caused by Bad-mKhris$m$ Go-Nad.
\end{abstract}

Keywords: Bad-mKhris-mGo-Nad; Bloodletting; Humor; gSo-ba rig-pa.

\section{INTRODUCTION}

\section{What is Bad-mKhris-mGo-Nad?}

Bad-mKhris-mGo-Nad is one type of headaches among 14 other types mentioned in gSo-ba-Rig-pa text, which is caused by the imbalance of bile and Phlegm humor. gSo-ba-Rig-pa defines the headaches in two categories; primary headaches and secondary headaches. The primary headache which consists of 8 types: rLung, mKhris, Thrak, Bad-Ken, Bad-mKhris, Bad-rLung, rLung-mkhrisand Sen. There are six secondary headaches; $M a$ Lung, sMen-Dras, gLog-Pa-Chan, sTra-Je, Chi-TherandsTrakar. In total there are 14 types of headaches. Bad-mKhris-mGoNad is one among the principal headaches ${ }^{1}$.

"In modern medicine, before antibiotics were introduced, bloodletting was used to treat serious ailments and infections such as pneumonia"'.

\section{Corresponding author:}

Karma Ugyen

ugyensangay@gmail.com
Bloodletting Therapy (BLT) is the last and final treatment for any heat-related disorder. This therapy is also the final and effective therapy for releasing heat from veins. The human body has 77 bloodletting points from which extraction of blood is done according to the condition of diseases. According to the Annual Health Bulletin (AHB), 289 patients had availed the services of bloodletting therapy from different TM hospitals across the country in 2017. The highest numbers of bloodletting was conducted at National Traditional Medicine Hospital (NTMH ) which was $211^{3}$. The prime objective of the study is to observe the effectiveness of bloodletting among diagnosed cases of $\mathrm{Bad}$ mKhris-mGo-Nad.

\section{METHODS}

\section{Study design}

It is an observational prospective study conducted at the National Traditional Medicine Hospital (NTMH) Kawangjansa, Thimphu, Bhutan, for a period of 6 months from 28 $8^{\text {th }}$ June to December 2018 after prior approval from the Research Ethic Board, Ministry of Health, vide approval letter No. REBH/Approval/2018/031. 


\section{Sample size}

The patients reporting to NTMH, Kawajangsa fulfilling inclusion criteria such as normal hemoglobin: Male: $>13.8-17.2 \mathrm{~g} / \mathrm{dl}$ and Female: $>12.1-15.1 \mathrm{~g} / \mathrm{dl}$, age range of 17 to 60 , without other serious pre-existing conditions like diabetes or be dependent on anticoagulant drugs and all diagnosed cases of Bad-Mkhris$m$ Go-Nad patients referred to Invasive Therapy Department were included. The diagnose of Bad-mKhris-mGo-Nad was made by traditional Medicine physician. At no point of the study did the researcher were involved in diagnosis of the patients. The same physician also performed the bloodletting therapy, which was observed by the researcher and interviewed according to the study protocol.

Though 45 participants were enrolled for the study, 14 of them couldn't complete the mandatory follow up as per the study protocol. Therefore, 31 participants who completed all the mandatory follow up were included; 23 females and 8 males between the age of 20 to 60 years, and most of the participants $(51.6 \%)$ had no formal education, followed by $19.35 \%$ with monastic education as mentioned in Figure 2. Those participants were interviewed using structure schedule by the principle investigator.

Bloodletting appointments are available from Tuesday to Friday. However, the patients were advised to avoid the

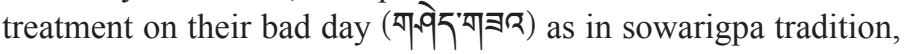
the body part where bloodletting therapy is to be performed must be free from life force or the vital energy (ㄱ) . The energy or $b L a$ is the life force in our body, which is believed to be always on the move, it moves in clockwise direction in females and anticlockwise in male 4 . So, it is necessary to find the exact site of the $b L a$ as per the waxing and waning phases of the moon and the therapy should not be conducted on the $8^{\text {th }}, 15^{\text {th }}$ and the $30^{\text {th }}$ of the lunar calendar.

If the therapy takes place during this period, it may cause more harm than benefit ${ }^{4}$. The patient was prescribed with the decoctions called Dresumthang, since this medicine has the potency to classify the impure and pure blood ${ }^{5}$.

\section{Bloodletting procedure}

The actual procedure involves immobilization of the body part where the bloodletting to be performed, followed by standard incision technique as per the Standard Operating Procedure in Sowa Rigpa ${ }^{6}$ blood analysis, and the standard amount of the blood to be withdrawn as per the SOP of bloodletting. Once the bloodletting procedure is over, the physician ensures that post procedure precautions are taken care and relevant advices are given to avoid the post therapeutic complication ${ }^{7}$. The standard amount of blood to be let out from Tshoksang is set to 5 to $20 \mathrm{ml}$ depending on the severity of the disease and the immunity of the patient $^{8}$.

For every participant, data collection was carried out on three occasions; Firstly, before conducting bloodletting therapy,

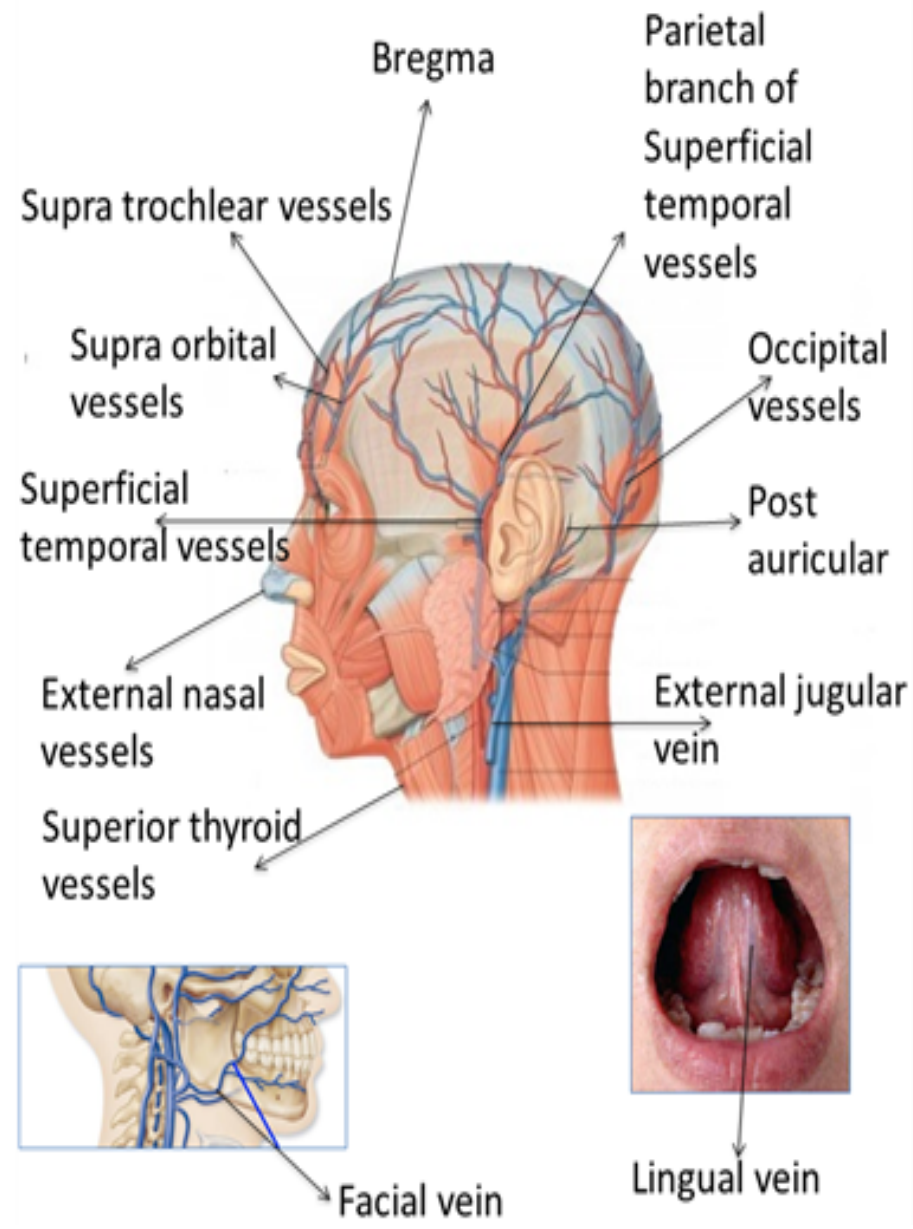

Figure 1. 21 bloodletting points on head

Second on $10^{\text {th }}$ day of post therapy and last one $30^{\text {th }}$ day post procedure.

The participants were asked regarding the frequency of headache in the following scale: daily, weekly, monthly, twice a month, thrice a month or no pain. The duration of the pain was measured in minutes and hours. The pain severity was measured on 1-10 of color coded numerical scale ${ }^{9}$. The post-treatment follow-up after 10 days and 30 days were mainly focused on the three main parameters (frequency, duration and severity of headache) and an association was also made to compare the result of the pre and post findings.

\section{RESULTS}

This study was carried out to measure the effect of Bloodletting therapy in treating Bad-mKhris-mGo-Nad. Most of the respondents were females with $74.19 \%$ and only $25.85 \%$ of the respondents were males. $72 \%$ of the sample $(n=31)$ were reported to be pre-diagnosed with Migraine condition from the allopathic hospital. Three parameters (frequency, duration, severity) 


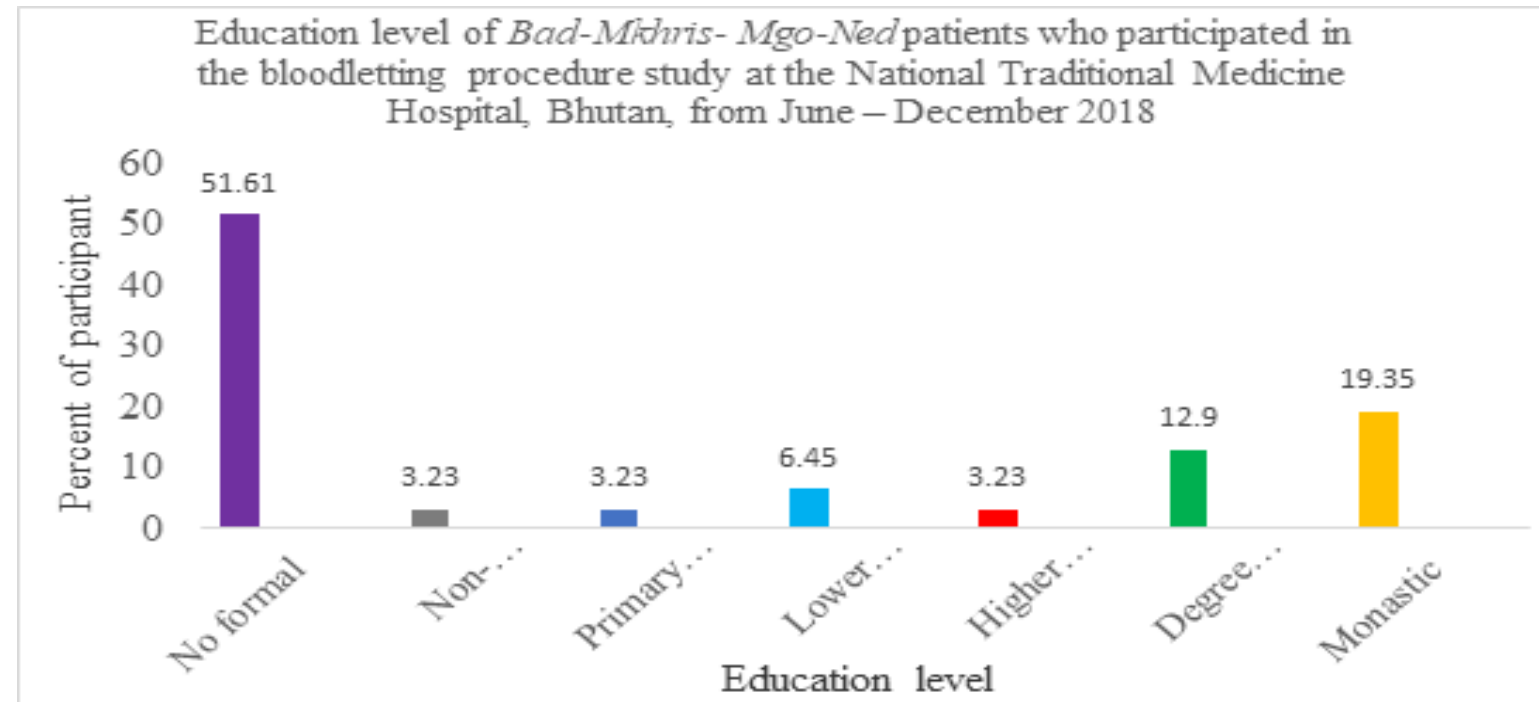

Figure 2. Education level of respondents

Table 1. Association test of pre and post intervention

\begin{tabular}{|c|c|c|c|c|c|c|c|c|}
\hline \multirow{2}{*}{ Characteristics } & \multicolumn{2}{|c|}{ Before Bloodletting } & \multicolumn{2}{|c|}{ After 10 days } & \multirow{2}{*}{$\begin{array}{l}p \text {-value } \\
\text { (2-tail)1 }\end{array}$} & \multicolumn{2}{|c|}{ After 30 days } & \multirow{2}{*}{$\begin{array}{l}p \text {-value } \\
\text { (2-tail)2 }\end{array}$} \\
\hline & $(n=31)$ & $\%$ & $(n=31)$ & $\%$ & & $(n=31)$ & $\%$ & \\
\hline \multicolumn{9}{|l|}{ Frequency of headache } \\
\hline $\begin{array}{l}\text { Thrice a month or } \\
\text { more frequent }\end{array}$ & 28 & 90.32 & 26 & 83.87 & & 13 & 41.94 & \\
\hline Twice a month or & & & & & 0.481 & & & $0.000 *$ \\
\hline $\begin{array}{l}\text { less frequent or no } \\
\text { pain }\end{array}$ & 3 & 9.68 & 5 & 16.13 & & 18 & 58.06 & \\
\hline \multicolumn{9}{|l|}{ Duration of headache } \\
\hline $\begin{array}{l}<30 \text { Minutes or } \\
\text { No pain }\end{array}$ & 1 & 3.23 & 10 & 32.26 & $0.003 *$ & 13 & 41.94 & $0.000 *$ \\
\hline$>30$ Minutes & 30 & 96.77 & 21 & 67.74 & & 18 & 58.06 & \\
\hline
\end{tabular}

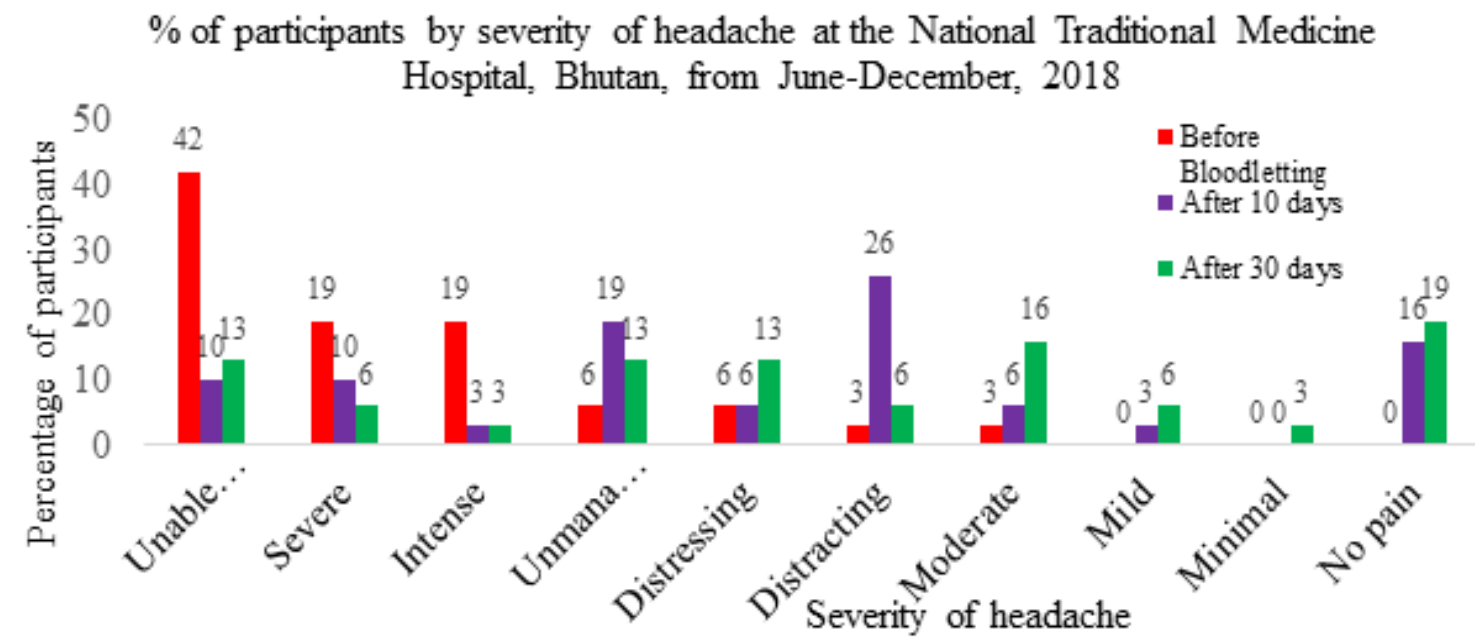

Figure 3. Severity of the headache 
were used to assess the effectiveness of gSo-ba-Rig-pa based bloodletting therapy. The result shows that the bloodletting could be effective in reducing the frequencies, duration, and severity of the pain. The association test between pre and post of 10 days and pre and post of 30 days of bloodletting was done by using Mid-P test in Open Epi. It was observed statistically significant at $p<0.05$.

\section{Statistical result of the Benefits of bloodletting procedure among Bad-mKhris-mGo-Nad patients}

Descriptive statistical analysis was used to describe data using mean, count, percentage, through tables and figures. Inferential statistical analyses could not be conducted due to the small sample size. However, Mid-p exact test of association was done in Open Epi by regrouping the frequencies. Mid-P exact test of association between before and after 10 days of bloodletting and a Mid-P exact test of association between before and after 30 days of bloodletting was conducted, and the result was Statistically significant at $p<0.05$ as presented in the Table 1 and Figure 3.

The above table shows that the bloodletting procedure has helped in reducing the frequency, duration and severity of headaches. All three parameters have declined after each consecutive follow up periods(on Tenth and thirtieth day).

\section{DISCUSSION}

Bloodletting therapy is effective in reducing the frequency, duration and severity of headache caused by Bad-mKhris-mGo$\mathrm{Nad}$. The finding emphasizes that, the bloodletting therapy on Tshoksang reduces the pain duration and frequencies of headache in patients with Bad-mKhris-mGo-Nad.

Since the main cause of Bad-mKhris-mGo-Nad is bile and phlegm disorder, bloodletting helps to reduce the burning hot nature of the bile humor, thus creating a proper counterbalance between the three humors. Some studies in the past claimed that reduction in pain duration is connected with placebo effect but according to the philosophy of gSo-ba-Rigpa, in order to maintain a pain-free body there should be a good balance of all three humors in the body ${ }^{10}$.

In Chinese Medicine, the pain in migraine is a result of blood stagnation. This can be only treated through rectifying the blood ${ }^{11}$. One of the benefits of bloodletting mentioned gSoba-Rigpa is relieving severe pain as sustained by this study. This result is also supported by a similar study published in the Journal of International Academic Research for Multidisciplinary ${ }^{12}$.

Despite all the claims made elsewhere in world, there are no studies in Bhutan to furnish this claim. Thus, this study can play a vital role in the history of Traditional Medicine in a quest towards institution of evidence-based practice in gSo-ba-Rigpa in Bhutan.

However, with this study has its own limitation, the sample size was small and as a result the findings from this study cannot be generalized to the larger population. In addition, there were extremely limited literature materials related to bloodletting. This again limited the ability to relate the findings from this study with others, which would have given more clarity and understanding our findings.

\section{CONCLUSIONS}

The result of the study shows that bloodletting therapy may be effective in reducing the frequencies, duration, and severity of the pain in Bad-mKhris-mGo-Nad. However, there is need to conduct studies in the future with bigger sample and more sites to be able to generalize the findings to population.

\section{REFERENCES}

1. Tshewang K. Kem-Drel, $2^{\text {nd }}$ rev.ed . Menrampa lozang Chophel, Dharamsala: Documentation and Publication Department. Men-tse-khang: Tibetan Medical and Astrological Institute of H.H the Dalai Lama; 1997. 216 p.

2. Hitti M. Bloodletting's Benifits [Internet]. 2004 [cited 2018 Apr 21]. [Full Text]

3. MoH. Annual Health Bulletin 2018. Thimphu, Bhutan: Ministry of Health; 2018. [Full Text]

4. Men-Tsee-Khang. Fundamental of tibetan medicine. $5^{\text {th }}$ rev. ed. HPl, Dharamsala: Men-Tsee-Khang; 2009. 62-63 p.

5. Tashi T, Dorji Y, Gembo. Therapy guideline for traditional medicine practitioner. $1^{\text {st }}$ rev.ed. Tshering P, Dorji T, Dorji J, Tashi D, Gylek K, PRU Sherub TK, editor. Thimphu: NTMH; 2008. 44 p.

6. DTMS. SOP. Thimphu, Bhutan: Department of Traditional medicine; 2012. p. 32-7.

7. Thokmay P, Wangdue P, SD. The subsequent tantra in english. $2^{\text {nd }}$ rev.ed. Thokmay P, Tshering T, Jamyang LN D, Choying T, editors. HPl, Dharamsala: Men-Tsee-Khang; 2015. 204-221 p.

8. Gyelwa. Chedchok Taar gi laklen kensel adrur rai melong. $1^{\text {st }}$ rev.ed. Tshognen: Tshognen Merig Petruenkhang; 2012. $1-133 \mathrm{p}$.

9. Patient F. Pain scale for people in chronic pain [Internet]. 2017 [cited 2018 Feb 21]. [Full Text]

10. Tsenam T. Sorig Juezhi Drelchen Drangson Zhelung book 1. 1st rev.ed.Tibet: Sithoren merig petrunkhang; 2000.

11. McCann H. Pricking the Vessels - Bloodletting therapy in Chinese medicine China. Institute for Classical Asian Medicine 300 Madison Avenue, Madison, NJ 07940; 2008. 29-38 p. [Full Text]

12. Shahrakivahed A, Firouzkouhi M, Moushtagh Z. The effect of bloodletting on migraine headache. [internet]. Iran: 2014 Sept. [Full Text] 
AUTHORS CONTRIBUTION

Following authors have made substantial contributions to the manuscript as under:

KU: Concept, design, data collection and analysis, manuscript writing and review.

KT: Design, data collection and analysis, manuscript writing and review

TG: Design, data collection and analysis, manuscript writing and review

Author agree to be accountable for all respects of the work in ensuring that questions related to the accuracy and integrity of any part of the work are appropriately investigated and resolved.

\section{CONFLICT OF INTEREST}

None

GRANT SUPPORT AND FINANCIAL DISCLOSURE

None 